\title{
Fibrome ossifiant mandibulaire associé à une canine incluse
}

\author{
Hajer Hentati ${ }^{1,}{ }^{*}$, Leila Njim²${ }^{2}$, Mohamed-Mekki Fehri ${ }^{1}$, Abdelfatteh Zakhama ${ }^{2}$ \\ Jamil Selmi ${ }^{1}$ \\ ${ }^{1}$ Service de Médecine et Chirurgie buccales, Clinique universitaire de Médecine dentaire, Monastir, Tunisie \\ ${ }^{2}$ Service d'Histopathologie, Centre hospitalo-universitaire Fattouma Bourguiba, Monastir, Tunisie
}

(Reçu le 21 janvier 2013, accepté le 8 février 2013)

Mots clés :

fibrome ossifiant /

dent incluse /

lésion fibro-osseuse

Key words: ossifying fibroma / impacted tooth / fibro-osseous lesion

\begin{abstract}
Résumé - Une patiente âgée de 25 ans a consulté pour une tuméfaction mandibulaire, parasymphysaire gauche, non douloureuse, d'évolution lente. À l'examen clinique, des anomalies dentaires ont été révélées, en particulier la persistance de la 73 sur l'arcade et l'absence de la 33. L'examen radiologique a montré une image mixte bien limitée juxtaposée à la 33 incluse. Le traitement a comporté l'extraction de la 33, l'exérèse complète de la lésion et le curetage de la cavité osseuse. L'examen histopathologique a conclu au diagnostic de fibrome ossifiant. L'association de cette tumeur à une dent incluse est exceptionnelle.
\end{abstract}

\begin{abstract}
Mandibular ossifying fibroma associated with impacted canine. A 25-year-old woman consulted for a bone swelling on her left mandible. History revealed that the mass increases progressively without any pain. Intraoral examination found, besides the bone swelling, dental anomalies particularly the persistence of 73 on the arch and the absence of 33. Radiographically, the lesion was well defined, non-homogeneous with central radioopaque focus. The 33 was impacted and juxtaposed to the lesion. The patient was treated with surgical excision of the lesion, curettage of the bone and extraction of the 33. A histopathological diagnosis of ossifying fibroma was made. The association of ossifying fibroma with impacted tooth is exceptional.
\end{abstract}

Le fibrome ossifiant est une tumeur odontogène bénigne rare. Il est constitué par un mélange de tissu fibreux et de matériel minéralisé. Cliniquement, il se traduit par une tuméfaction osseuse qui peut être associée à des signes dentaires tels que déplacement des dents ou résorption radiculaire. Ce travail rapporte un nouveau cas de fibrome ossifiant associé à une canine incluse.

\section{Observation}

Une patiente, âgée de 25 ans, sans antécédents pathologiques particuliers, a consulté pour une tuméfaction vestibulaire inférieure para-médiane gauche, ferme, évoluant lentement et progressivement depuis deux ans, sans douleurs ni troubles de la sensibilité. L'examen clinique montrait une asymétrie faciale secondaire à la tuméfaction de la région parasymphysaire gauche. La peau en regard était normale. L'examen endobuccal retrouvait une tuméfaction osseuse vestibulaire s'étendant de la ligne médiane à la 34, dure, non douloureuse à la palpation (Fig. 1). La muqueuse de recouvrement était normale. Seule la 36, qui n'était pas en rapport avec la lésion, était nécrosée après récidive de la carie sous une obturation défectueuse. L'examen dentaire a mis en évidence les anomalies suivantes : persistance de la 73 sur l'arcade, absence clinique de 33 et de 12, 22, 18 et 28 sans notion d'extraction et enclavement de 38 et 48 . Il existait des diastèmes entre les dents du groupe incivo-canin supérieur et entre 41 et 42 ; ces deux dernières étant égressées.

L'orthopantomogramme montrait une image mixte, bien circonscrite s'étendant de la 31 à la 34 ; la face mésiale de la 33 incluse était juxtaposée à la lésion. Les racines de 32 et 34 étaient divergentes, celle de 73 présentait une résorption. On notait également la présence de 18 et 28 incluses et l'absence (agénésie) de 12 et 22 (Fig. 2).

Sous anesthésie loco-régionale, une intervention chirurgicale par voie d'abord vestibulaire a été réalisée. Le décollement d'un lambeau muco-périosté a mis en évidence une corticale vestibulaire déformée et amincie. La lésion était constituée par une masse centrale dure, de couleur blanc nacré, mesurant $1,5 \mathrm{~cm}$ dans son grand axe, et par une partie périphérique

\footnotetext{
*Correspondance : hajer_hentati@yahoo.fr
} 


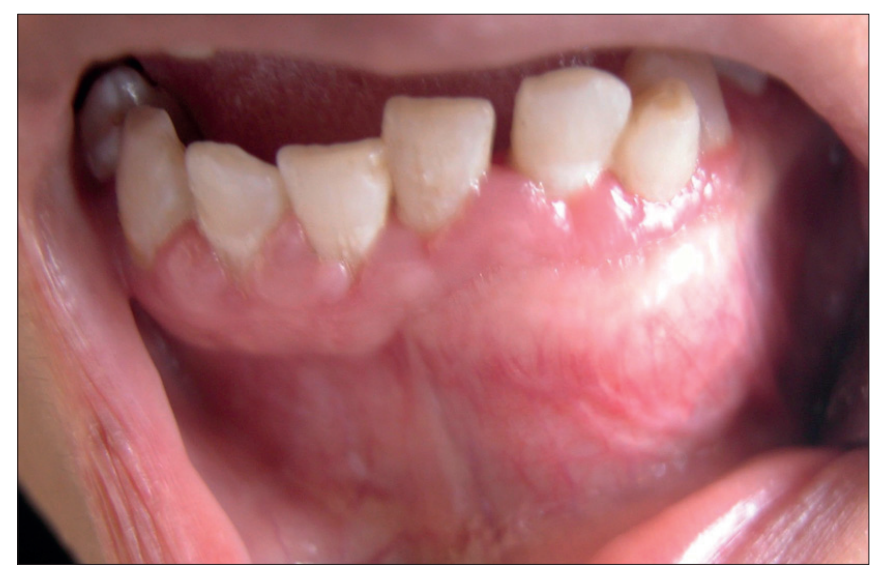

Fig. 1. Vue endobuccale : tuméfaction vestibulaire. Fig. 1. Oral view: vestibular swelling.

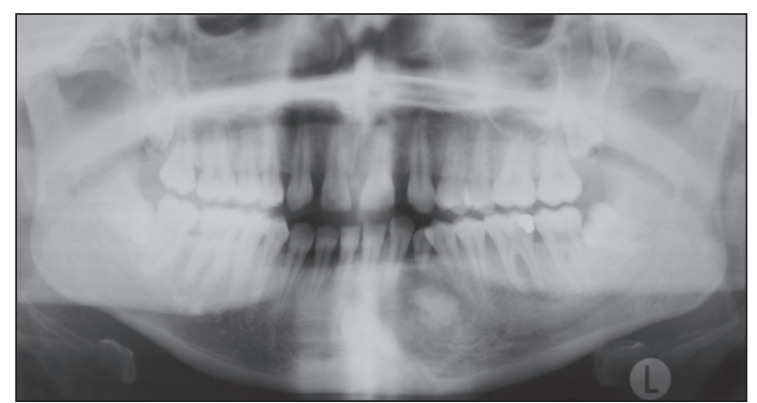

Fig. 2. Orthopantomogramme : image mixte et 33 incluse. Fig. 2. Orthopantomogram: mixed image and 33 impacted.

moins dure, mesurant $1,2 \mathrm{~cm}$ dans son grand axe. L'exérèse a été assez facile car il y avait un plan de clivage. Elle a été complétée par un curetage de la paroi osseuse et l'extraction de la 33 (Fig. 3). Les suites opératoires ont été simples.

L'examen histopathologique du fragment central montrait un tissu osseux sous forme de travées épaisses, séparées par des espaces fibreux bien vascularisés ; ce dernier était entouré par du tissu fibreux riche en fibroblastes, parsemé de travées osseuses néoformées et de foyers minéralisés arrondis rappelant le cément. Le deuxième fragment avait un aspect comparable à la périphérie du précédent (Fig. 4). Le diagnostic histopathologique de fibrome ossifiant a été retenu.

La patiente qui était peu coopérante, a refusé une prise en charge orthodontique et prothétique.

\section{Commentaires}

Selon la dernière classification histologique des tumeurs odontogènes de l'organisation mondiale de la santé (OMS) établie en 2005, le fibrome ossifiant (F0) appartient au groupe de lésions en rapport avec l'os. Le fibrome cémentifiant (FC)

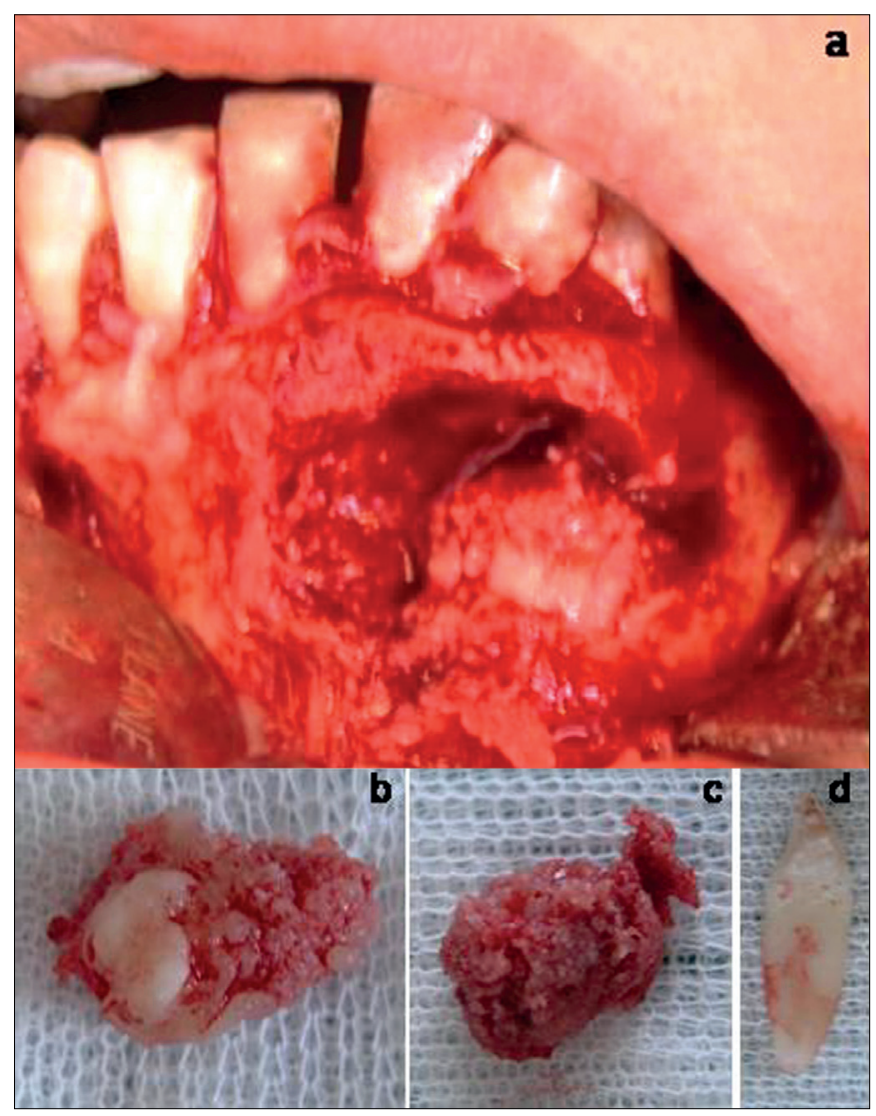

Fig. 3. Exérèse de la tumeur ; $3 a$. vue peropératoire ; 3b. fragment central ; 3c. fragment périphérique ; 3 d. 33 extraite.

Fig. 3. Excision of the tumor; $3 a$. intraoperative view; $3 b$. central fragment; 3c. peripheral fragment; $3 d .33$ removed.

et le fibrome cémento-ossifiant (FCO) sont des termes synonymes de F0. Le fibrome ossifiant trabéculaire juvénile (FOTJ) et le fibrome ossifiant psammomateux juvénile (FOPJ) sont considérés comme deux variantes histologiques; les formes juvéniles sont dites aussi actives ou agressives [1]. Dans la classification précédente de l'OMS (1992), le FCO constituait à lui seul le sous-groupe des tumeurs ostéogénes appartenant au groupe des tumeurs et autres lésions en rapport avec l'os. Il est constitué de tissu fibreux contenant des quantités variables d'un matériel minéralisé ressemblant à de l'os et/ou à du cément, ce qui a permis d'individualiser deux variétés, le F0 et le FC [2].

Le F0 se développerait à partir des cellules mésenchymateuses multipotentes du ligament parodontal qui sont capables de former de l'os et du cément [3]. Des cellules ectopiques du ligament seraient à l'origine des localisations extragnathiques [4]. Le traumatisme a été également considéré comme un agent étiologique [5].

C'est une tumeur relativement rare [6] qui constitue moins de $0,1 \%$ de l'ensemble des tumeurs odontogénes [7]. Dans les 


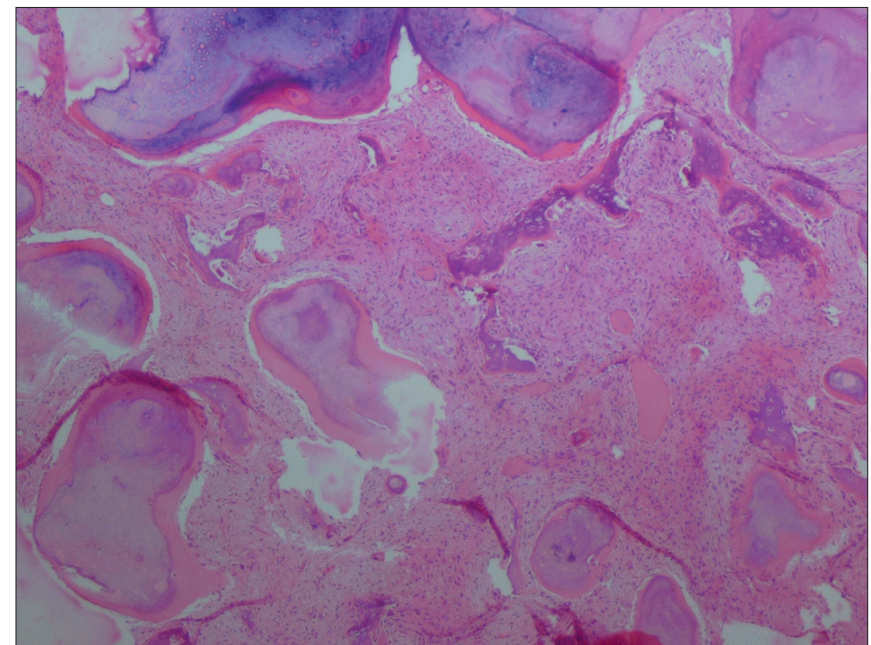

Fig. 4. Aspect histologique: tissu fibreux parsemé de travées osseuses et de sphérules cémentoïdes.

Fig. 4. Histologic feature: fibrous tissue with lamellar bone and spherules of cementum-like material.

formes juvéniles, le FOPJ est plus fréquent que le FOTJ [8]. Le F0 survient, le plus souvent, entre la deuxième et la quatrième décennies avec une moyenne d'âge qui varie selon le sous-type histologique : 35 ans pour le FO conventionnel, 20 ans pour le FOPJ et entre 8 ans et demi et 12 ans pour le FOTJ [1]. Les femmes sont plus atteintes que les hommes [9]. La localisation préférentielle varie selon le sous-type histologique : la région prémolo-molaire de la mandibule pour le F0 conventionnel [10], les os cranio-faciaux extragnathiques, particulièrement les os périorbitaires, le frontal et l'éthmoïde pour le FOPJ [11], et le maxillaire pour le FOTJ [8]. Des localisations rares du F0 ont été rapportées : sinus éthmoïdal, sinus maxillaire, sinus sphénoïdal et exceptionnellement l'os temporal avec extension intracrânienne [4].

Le F0 est souvent unique ; cependant, il existe des formes multiples [10]. Elles ont été associées à des anomalies hormonales telle que l'hypercalcémie liée à l'hyperparathyroïdie. Elles ont été également rapportées en association avec le syndrome hyperparathyoïdie - tumeur mandibulaire [9]. Les lésions fibroosseuses de la mandibule ou du maxillaire associées à ce syndrome ont tendance à régresser lentement après traitement de l'hyperparathyroïdie. Celles qui ont été réséquées chirurgicalement en l'absence de régression suffisante, ont été diagnostiquées histologiquement comme $\mathrm{FO}$ ou FC ou FCO [12]. Les formes multiples sporadiques sont exceptionnelles [9]. Canger et al. ont rapporté un cas familial de FO avec deux localisations chez le père (une maxillaire et une mandibulaire) et une localisation unique mandibulaire chez la fille diagnostiquée 3 mois plus tard [3]. Le F0 peut être associé exceptionnellement à un granulome central à cellules géantes [13] ou à un kyste osseux anévrysmal [8].
En général, la tumeur est asymptomatique jusqu'à ce qu'elle se traduisent par une tuméfaction et une déformation [14]. L'évolution est souvent lente, parfois rapide et extensive [5], entre autres dans les formes juvéniles [15]. Les petites lésions, asymptomatiques, sont découvertes lors d'examens radiologiques dentaires de routine [6]. Les douleurs et les paresthésies sont rares [10]. Les lésions plus étendues provoquent une déformation faciale progressive avec des signes d'accompagnement, variables selon la localisation [16] : obstruction nasale, épistaxis [11], signes dentaires (déplacement et mobilité). Une extension intra-orbitaire peut provoquer une exophtalmie, une diplopie, une limitation des mouvements oculaires et la perte progressive de la vision par atrophie du nerf optique [7] ; une extension intracrânienne des signes neurologiques par compression [4] et rarement une méningite [6].

Radiologiquement, le F0 donne une image bien limitée qui peut apparaître complètement radiotransparente, uni ou multiloculaire, avec ou sans liseré d'ostéo-condensation, ou le plus souvent mixte avec une (des) opacité(s) centrale(s) $[6,10,17]$. L'aspect radio-opaque avec un liseré radiotransparent périphérique est rarement observé [6]. La densité de l'image dépend du degré de minéralisation qui évolue avec l'âge de la lésion $[6,11]$. Dans les régions dentées, la croissance continue de la lésion peut engendrer des divergences et/ou des résorptions radiculaires [3] ainsi que des déplacements dentaires [18].

Dans le cas rapporté, le F0 présente la particularité d'être associée à une canine incluse. La présence d'une tuméfaction osseuse et d'une radio-opacité centrale fait suspecter une lésion ancienne. L'examen tomodensitométrique permet de visualiser une coque osseuse autour de la tumeur sans érosion de la corticale. En imagerie par résonnance magnétique, l'intensité du signal en T1 et T2 rehaussé dépend de certains facteurs tels que la quantité de l'os trabéculaire et le degré de cellularité [17].

Macroscopiquement, le F0 se présente comme une lésion ferme, bien limitée [1], parfois encapsulée [6]. Microscopiquement, il comporte deux composantes : une composante minéralisée et du tissu fibreux. La composante minéralisée prend un aspect variable, ressemblant à des structures osseuses et/ ou cémentaires; la distinction histologique entre tissu ostéoïde et tissu cémentaire est souvent difficile [15]. Les structures ressemblant au cément ne sont qu'une variante d'os et ce sont les mêmes cellules progénitrices qui produisent ces deux tissus [6]. La cellularité du tissu fibreux est très variable : il $y$ a des zones cellulaires et d'autres peu cellulaires, voire acellulaires [1]. Le FOTJ comporte un stroma hypercellulaire contenant des bandes de tissu ostéoïde cellulaire et des trabécules d'os immature. Le FOPJ se caractérise par la présence d'ossicules ressemblant aux corps psammomateux [1].

Le principal diagnostic différentiel du F0 est la dysplasie fibreuse (DF). En dehors des cas typiques, ces deux lésions peuvent présenter des caractéristiques radiologiques et histopathologiques similaires. Cependant, l'évolution de ces deux 
affections est bien différente et elles nécessitent, par conséquent, des thérapeutiques adaptées. Toyosawa et al. ont proposé deux techniques pour affiner le diagnostic différentiel entre ces deux affections. La première est basée sur l'étude immunohistochimique de l'ostéocalcine dont l'expression serait faible dans la matrice osseuse de 5 spécimens de F0 et forte dans celle de 9 spécimens de DF ( 5 localisations gnathiques, 4 extragnathiques). La deuxième technique consiste à réaliser l'étude moléculaire des mutations sur le codon arginine 201 $\left(\operatorname{Arg}^{201}\right)$ du gène codant la sous-unité alpha de la protéine stimulatrice G (GNAS), qui serait un marqueur de la DF extragnathique. Les mutations étaient présentes dans tous les cas de DF étudiées et absentes pour les cas de F0 [19]. Le diagnostic différentiel se pose également avec la dysplasie osseuse [2], la dysplasie cémento-osseuse focale [6], la tumeur épithéliale odontogénique calcifiée [15], l'ostéomyélite sclérosante chronique, l'ostéome ostéoïde et l'ostéoblastome [5]. Quand le F0 est totalement radiotransparent, il peut prêter à confusion, selon sa localisation, avec un granulome péri-apical [5], un kyste dentigère [18], une mucocèle ou tout autre lésion radiotransparente [16] (kyste osseux solitaire, tumeur odontogène, kératokyste, améloblastome, granulome central à cellules géantes, myxome, tumeur odontogène adénomatoïde) [5].

Le traitement du F0 consiste en une exérèse avec curetage de la cavité osseuse. L'exérèse, souvent facilitée par la présence d'un plan de clivage [5], semble plus difficile au maxillaire qu'à la mandibule [20], et pour les formes juvéniles que pour les formes conventionnelles [8]. Les F0 étendus ou récidivants sont traités par résection avec éventuellement reconstruction par greffe osseux [10]. Des complications per-opératoires ont été rapportées : hémorragie sévère à partir des vaisseaux alimentant le tissu tumoral [18], fracture de la mandibule au cours de l'exérèse d'un F0 mandibulaire [10]. La récidive après exérèse complète est rare [6] ; aucune transformation maligne n'a pas été décrite [8].

\section{Conflits d'intérêt : aucun}

\section{Références}

1. Slootweg PS, El Mofty SK. Ossifying fibroma (pp. 319-20). In: Barnes L, Eveson JW, Reichart P, Sidransky D. World health organisation classification of tumours. Pathology and genetics of head and neck tumours. IARC Press, Lyon, 2005.

2. Kramer IRH, Pindborg JJ, Shear M. World health organisation histological typing of odontogenic tumours, 2nd ed (pp. 27-8). Springer Verlag, Berlin Heidelberg, 1992.

3. Canger EM, Celenk P, Kayipmaz S, Alkant A, Gunhan 0. Familial ossifying fibromas: report of two cases. J Oral Sci 2004;46:61-4.

4. Kansal R, Sharma A, Gaikwad N, Mahore A, Goel A. Cementoossifying fibroma presenting as a posterior fossa mass lesion. Turk Neurosurg 2010;20:265-8.
5. Sanchis JM, Peñarrocha M, Balaguer JM, Camacho F. Cementoossifying mandibular fibroma: a presentation of two cases and review of the literature. Med Oral 2004;9:69-73.

6. Neville BW, Damm DD, Allen CM, Bouquot JE. Bone pathology (pp. 533-88). In: Neville BW, Damm DD, Allen CM, Bouquot JE. Oral and maxillofacial pathology. 2nd ed. WB Saunders, Philadelphia, 2002.

7. Gunaseelan R, Anantanarayanan P, Ravindramohan E, Ranganathan K. Large cemento-ossifying fibroma of the maxilla causing proptosis: a case report. Oral Surg Oral Med Oral Pathol Oral Radiol Endod 2007;104:e21-5.

8. El-Mofty S. Psammomatoid and trabecular juvenile ossifying fibroma of the craniofacial skeleton: two distinct clinicopathologic entities. Oral Surg Oral Med Oral Pathol Oral Radiol Endod 2002;93:296-304.

9. Ribeiro AC, Carlos R, Díaz KP, Gouvêa AF, Vargas PA. Bilateral central ossifying fibroma affecting the mandible: report of an uncommon case and critical review of the literature. Oral Surg 0ral Med Oral Pathol Oral Radiol Endod 2011;111:e21-6.

10. Ong $\mathrm{AH}$, Siar $\mathrm{CH}$. Cemento-ossifying fibroma with mandibular fracture. Case report in a young patient. Aust Dent J 1998;43: 229-33.

11. Eversole R, Su L, ElMofty S. Benign fibro-osseous lesions of the craniofacial complex. A review. Head Neck Pathol 2008;2:177-202.

12. Chen JD, Morrison C, Zhang C, Kahnoski K, Carpten JD, Teh BT. Hyperparathyroidism-jaw tumour syndrome. J Intern Med 2003;253:634-42.

13. Kaplan I, Manor I, Yahalom R, Hirshberg A. Central giant cell granuloma associated with central ossifying fibroma of the jaws: a clinicopathologic study. Oral Surg Oral Med Oral Pathol Oral Radiol Endod 2007;103:e35-41.

14. Pérez-García S, Berini-Aytés L, Gay-Escoda C. Ossifying fibroma of the upper jaw: report of a case and review of the literature. Med Oral 2004;9:333-9.

15. Kuta AJ, Worley CM, Kaugars GE. Central cementoossifying fibroma of the maxillary sinus: a review of six cases. AJNR Am J Neuroradiol 1995;16:1282-6.

16. Nasser MJ. Psammomatoid ossifying fibroma with secondary aneurysmal bone cyst of frontal sinus. Childs Nerv Syst 2009;25: 1513-6.

17. MacDonald-Jankowski DS. Fibro-osseous lesions of the face and jaws. Clin Radiol 2004;59:11-25.

18. Godt A, Gülicher D, Kalwitzki M, Kröber SM. Dislocation of an upper third molar by an ossifying fibroma - case report. J Craniomaxillofac Surg 2008;36:360-4.

19. Toyosawa S, Yuki M, Kishino M, Ogawa Y, Ueda T, Murakami S, Konishi E, Iida S, Kogo M, Komori T, Tomita Y. Ossifying fibroma vs fibrous dysplasia of the jaw: molecular and immunological characterization. Mod Pathol 2007;20:389-96.

20. Hekmatnia A, Ghazavi A, Saboori M, Mahzouni P, Tayari N, Hekmatnia F. A case report of cemento-ossifying fibroma presenting as a mass of the ethmoid sinus. J Res Med Sci 2011;16:224-8. 